\title{
Constrictive pericarditis - still an overlooked entity
}

\author{
Viviana Aursulesei ${ }^{*, 1,2}$, Alexandru Cozma ${ }^{1,3}$, Simina Bulughiana ${ }^{2}$, Irina Iuliana \\ Costache ${ }^{1,2}$
}

1“Gr. T. Popa" University of Medicine and Pharmacy lasi, Romania, ${ }^{2}$ Cardiology Department, University Emergency Hospital "Sf. Spiridon" Iasi, Romania, ${ }^{3}$ Internal Medicine Department, Army Hospital, lasi, Romania

\begin{abstract}
Constrictive pericarditis still remains a challenge for clinicians because it is a rare disease, with a long symptom-free period that can make it difficult to establish the relation between etiology and first clinical presentation. Multimodality imaging is the modern approach for diagnostic work-up and clinical management. In this context, we report a typical case of constrictive pericardial disease, first misconstrued as decompensated liver cirrhosis, and later unsuccessfully treated as congestive heart failure, without a clear etiology. By reporting this case, we also aimed to highlight the importance of some complementary diagnostic techniques in refining the preoperative assessment, in order to adequately address a patient to pericardiectomy, the only curative treatment.
\end{abstract}

Keywords: constrictive pericarditis, multimodality imaging, pericardiectomy

\section{Introduction}

In 1892, William Osler was the first to emphasize that "probably no serious disease is so frequently overlooked by the practitioner. Postmortem experience shows how often pericarditis is not recognized or goes on to resolution and adhesion without attracting notice" [1]. Despite the undeniable progress of diagnostic methods, constrictive pericarditis is still a challenge for clinicians, as it requires a high index of suspicion. Although a rare disease, it represents the late stage of an early and silent involvement of pericardium in many disorders, leading to dramatic effects on diastolic function. The difficult diagnosis, the poor understanding of the underlying cause

Received: February 2016; Accepted after review: March 2016; Published: March 2016.

${ }^{*}$ Corresponding author: Viviana Aursulesei MD $\mathrm{PhD}$, "Gr. T. Popa" University of Medicine and Pharmacy Iasi, Cardiology Department, University Emergency Hospital "Sf. Spiridon", 700111, lasi, Romania.

Email: aursuleseiv@yahoo.com and the high risk of pericardiectomy should be noted as main features of this severe disease.

\section{Case report}

A 36 year-old man was admitted for shortness of breath and typical signs of rightsided heart failure. The medical history of the patient started four years before the current admission, with a first diagnosis of liver cirrhosis, based on ascites, lower limb edema and umbilical hernia. The clinical hypothesis was reconsidered, since no signs of portal hypertension and no specific etiology were revealed. Furthermore, the jugular veins turgescence highly suggestive for a cardiac disease, ECG documentation of atrial fibrillation and the left ventricular ejection fraction (LVEF) of 40 percent allowed the following diagnosis: dilated cardiomyopathy, permanent atrial fibrillation and chronic heart failure (NYHA II class). Despite the medical treatment, the evolution was marked by 
repeated paracentesis for recurrent large ascites.

On current clinical presentation, the most important sign still was the large ascites. The physical examination also identified a postminimal pleurotomy scar, after draining a tuberculous pleurisy (hospitalized and treated for 6 months, 20 years before the current presentation) and other extensive postcombustional scarring on the left hemithorax, signs of chronic venous insufficiency of left lower limb, bilateral discrete edema, and turgescent jugular veins. There were no significant changes in hematological and plasma biochemical parameters. High voltage frontal chest X-ray demonstrated a normal-sized heart with pericardial calcification along its right inferior arch and a minimal right pleural effusion (Figure 1). The vein pressure was severely increased to $300 \mathrm{mmH}_{2} \mathrm{O}$. The key diagnostic method was echocardiography, emphasizing biatrial dilatation with normal-sized ventricles, thickened pericardium (Figure 2a), dilated inferior vena cava $(28 \mathrm{~mm})$, with diminished inspiratory collapse and mild-moderate pulmonary hypertension of $47 \mathrm{mmHg}$.

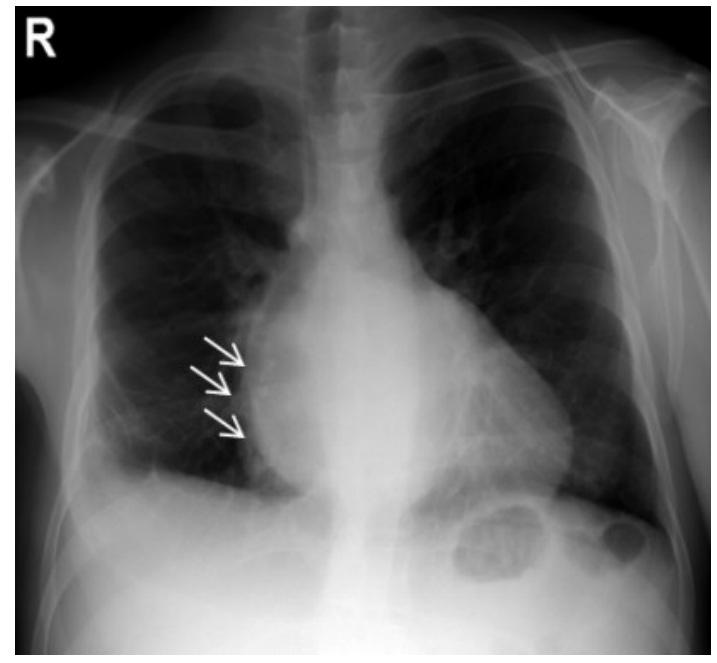

Fig. 1. Frontal chest X-ray. Cardio-thoracic index 0.50. Pericardial calcification on right inferior arch of the heart (arrows). Minimal right pleural effusion.

All these findings, along with the pathological changes of both left and right ventricular diastolic filling after inspiration led to the hypothesis of constrictive pericarditis (Figure 2b).

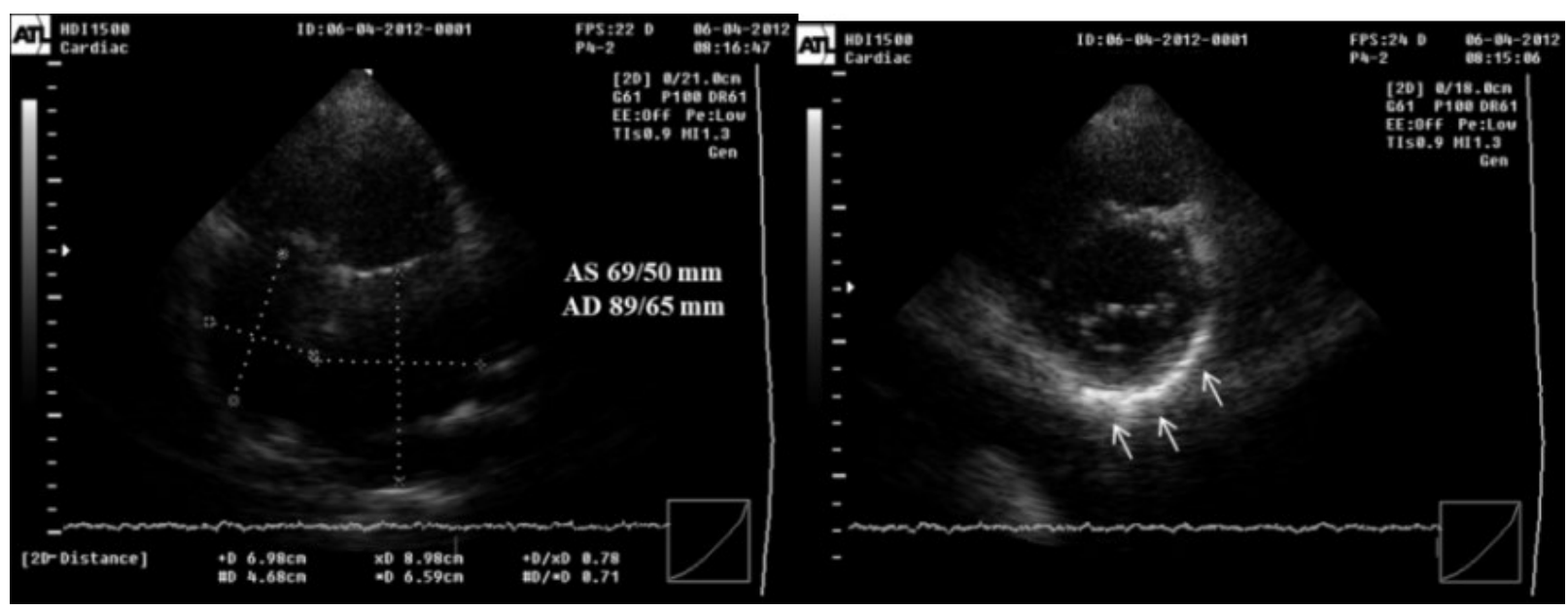

Fig. 2a. 2D-echocardiography. Apical 4-chamber view (left): biatrial dilatation. Parasternal short-axis view- mitral valve level (right): thickened pericardium $>2 \mathrm{~mm}$ (arrows). $A S=$ Left atrium; $A D=$ Right atrium 


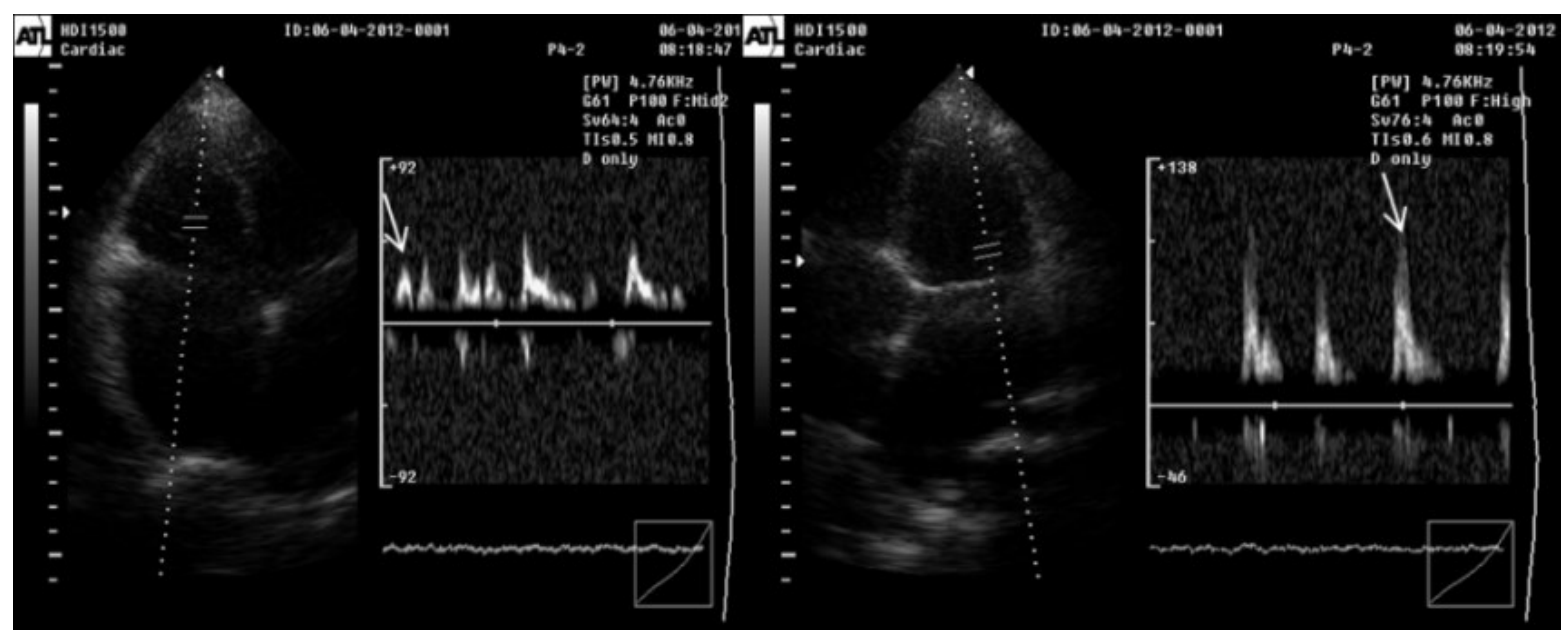

Fig. 2b. 2D-echocardiography. Apical 4-chamber view- echo pulsed wave Doppler. Pathological changes of mitral (left) and tricuspidian (right) flow velocities after inspiration.

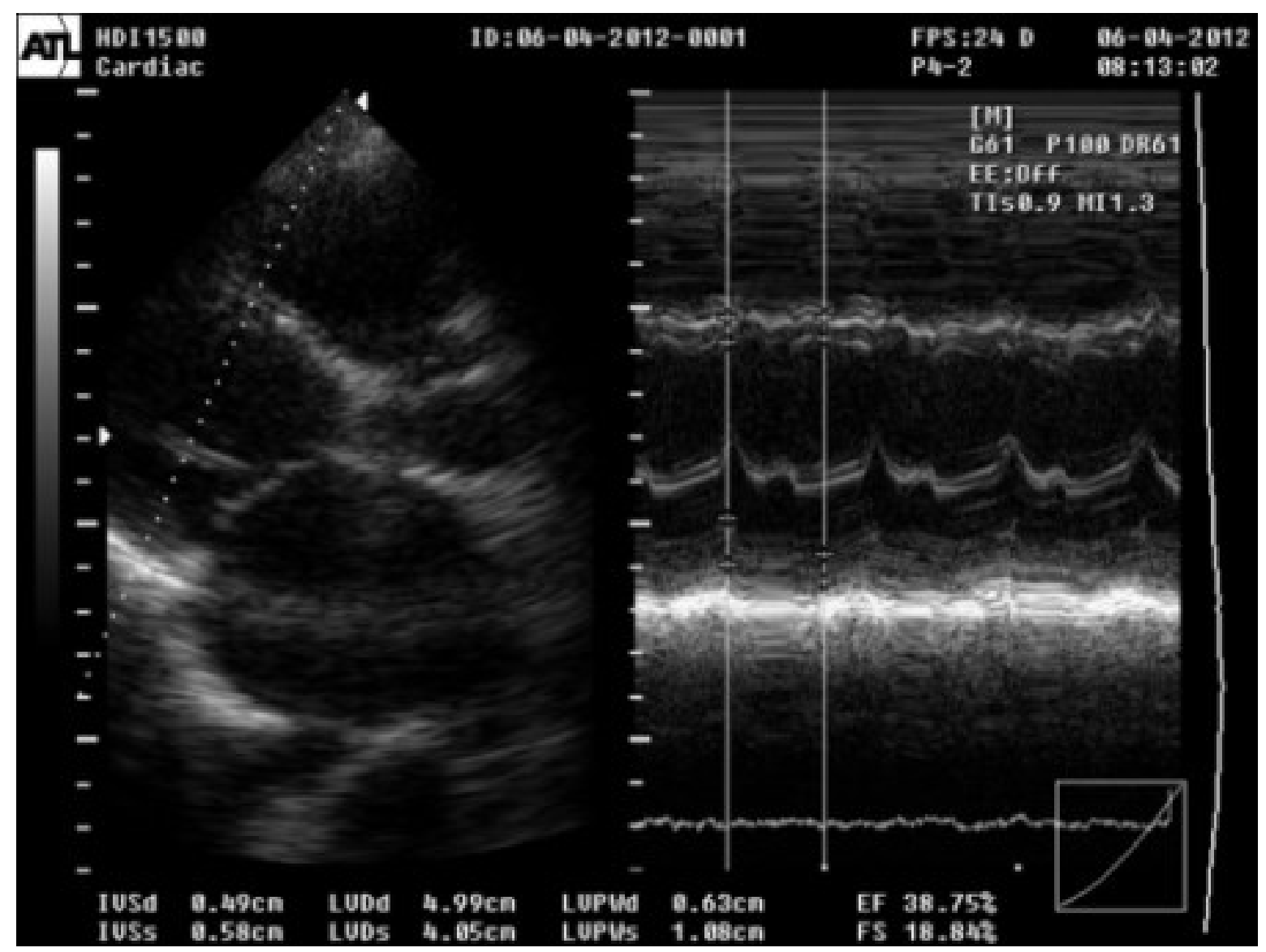

Fig. 2c. 2D-echocardiography. Parasternal long-axis view. M-mode (right): assessment of left ventricle ejection fraction using Teichholz method.

The depressed LVEF of 38.7 percent was considered a consequence of long-standing atrial fibrillation, but myocardial fibrosis inducing restriction also needed to be excluded (Figure 2c). The diagnosis of pericardial constriction was finally sustained by right cardiac catheterization, emphasizing equalization of telediastolic pressures in the 
right chambers of the heart and pulmonary artery $(16 \mathrm{mmHg})$ and "dip-and-plateau" pattern of the right ventricular pressure curve with $\mathrm{Yu}$ index of $44 \%$. The restriction was also excluded, as the difference between telediastolic ventricular curves pressure was less than $5 \mathrm{mmHg}$ (LV pressure 106/18 $\mathrm{mmHg}$, right ventricle pressure $36 / 16 \mathrm{mmHg}$ ) (Figure 3).

The patient was addressed to pericardiectomy, the only curative treatment for permanent constriction. For completing the preoperative assessment, a native thoracic CT was performed. Linear pericardial calcifications on right ventricle topography and areas of strong calcifications on right atrium topography (Figures $4 a$ and $4 b$ ), together with thin left ventricular walls $(<1 \mathrm{~cm})$, suggesting myocardial atrophy were recorded.

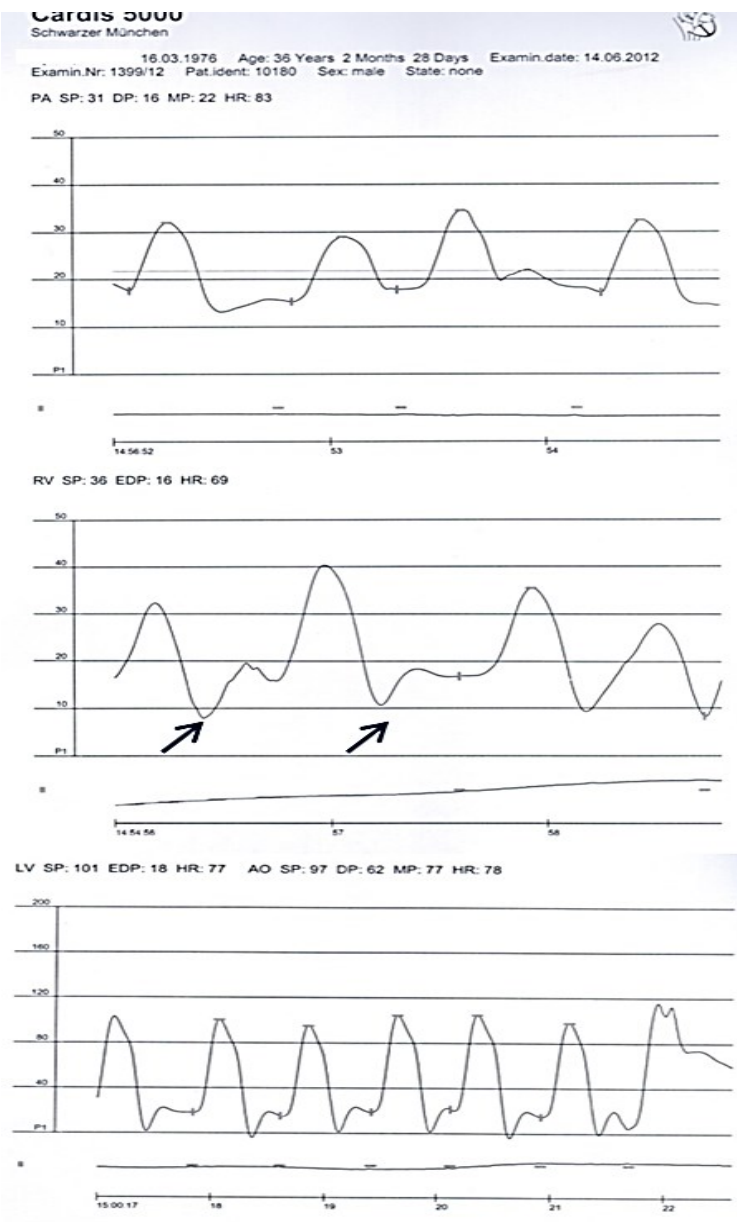

Fig. 3. Right cardiac catheterization. Barwell sign equalization of diastolic pressure (DP) in pulmonary artery and right chambers; dip and plateau pattern (arrows); left ventricle pressure curve.

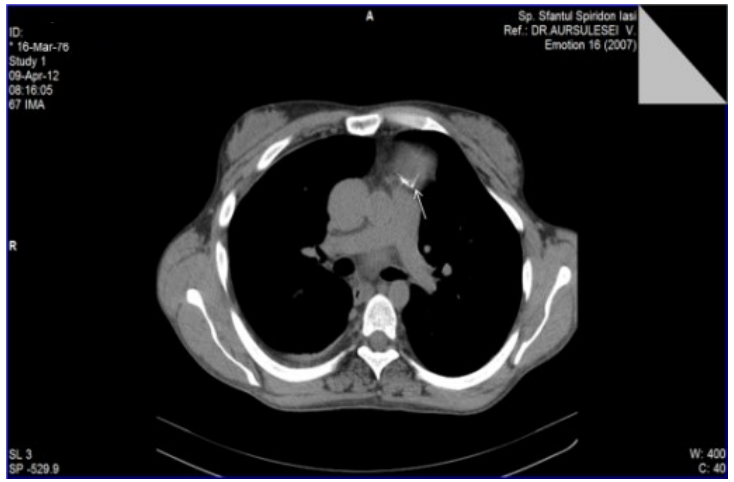

Fig. 4a. Native thoracic CT. Linear pericardial calcification on right ventricle topography.

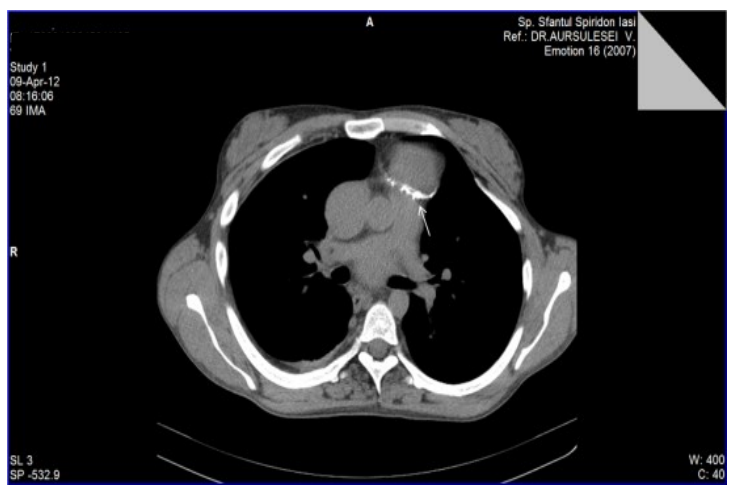

Fig. 4b. Native thoracic CT. Areas of calcified pericardium on right atrium topography.

The exam was completed with a CT pulmonary angiography to exclude pulmonary embolism, as a concurrent cause of right ventricular heart failure. A localized pericardiectomy through median sternotomy was successfully performed. Despite the postoperative worsening of cardiac hemodynamics, the long-term outcome was favorable. The medical treatment for left-sided heart failure and atrial fibrillation was continued together with chronic oral anticoagulation.

\section{Discussions}

We reported a typical case of constrictive pericarditis, frequently misconstrued as decompensated hepatic cirrhosis. From a clinical point of view, it must be noted that jugular veins turgescence in presence of socalled hepatic cirrhosis is most probably a right-sided heart failure. 
The final diagnosis of constrictive pericarditis still remains a challenge, because it is a rare disease, with a long symptom-free period that can make it difficult to establish the correlation between etiology and first clinical presentation. At the same time, our case is particular because the posttraumatic etiology, including non-penetrating thoracic injury is very rare, being reported on a few large historical clinical series [2, 3], while tuberculosis, sustained by the history of prior successful antituberculosis treatment for pleurisy, is still considered a major cause of pericardial disease in developing countries [4]. Secondly, the depressed LVEF imposed myocardial atrophy/fibrosis recognition, as it strongly influences cardiac mortality and morbidity following pericardiectomy.

Multimodality imaging is the modern approach for diagnostic work-up $[5,6]$. In constrictive pericarditis, chest $X$-ray shows a normal-sized heart, despite the clinical signs of congestive heart failure. Completing the examination with the high voltage technique, fibrous/calcified areas of the pericardium can be detected. CT, as a second-level imaging technique, is more accurate for assessing calcifications, pericardial thickening, degree and extension of pericardial involvement [4]. A key message is that fibrous/ calcified pericardium does not mean constriction, as $28 \%$ of constrictive pericarditis cases have normal pericardium [7].

In our case, CT technique was also useful to confirm myocardial atrophy, as it detected a thin interventricular septum $(<1 \mathrm{~cm})$ and stated no visualization of LV posterolateral wall. Compared with CT, cardiac magnetic resonance is probably the preferred technique to optimally assess constrictive pericarditis, as it equally provides morphological data and functional information about the hemodynamic consequences on cardiac filling [4]. Commonly used, transthoracic echocardiography is the first-line imaging test, providing all information for a complete and most cost-effective diagnosis. The most important finding is the pathological pattern of both left and right ventricular diastolic filling after inspiration. Given the irregular ventricular diastolic filling in the presence of atrial fibrillation, the diagnosis can be more difficult. In such cases, the final diagnosis can be confirmed by right cardiac catheterization. For our patient, the interventional technique was also useful to exclude the myocardial restriction.

Because it is still difficult to clearly separate constriction by restriction, a novel hemodynamic parameter named "systolic area index" has been successfully tested, with $97 \%$ sensitivity and $100 \%$ specificity for identifying patients with surgically proven constrictive pericarditis [9]. Noninvasive techniques, such as dual-source CT or 2D speckle-tracking echocardiography have also evolved, in order to improve the diagnostic accuracy and early addressing patients to surgical treatment [4, 10]. At the same time, the wrong selection of patients burdens the surgical procedure with a high risk of mortality [11]. However, the guidelines do not define clear indications for pericardiectomy. In order to solve this issue, some clinical predictors for better surgical outcome have been proposed, such as age, seric creatinine level, hyponatremia, hypoalbuminemia, NYHA class, reduced cardiac output, cachexia, etiology of pericarditis, abnormal LV contractility and relaxation abnormalities $[12,13]$.

\section{Conclusions}

By reporting this case, we aimed to highlight the importance of a complete diagnostic approach, in order to adequately address a patient to pericardiectomy.

At the same time, our case demonstrates the long, silent evolution of the disease, impacting the relation with its etiology, the nonspecific clinical presentation and thus, the difficult diagnosis. 


\section{References}

1. Soler-Soler J, Permanyer G, Sagristà-Sauleda J. Pericardial Disease: New Insights and Old Dilemmas. Dordrecht: Kluwer Academic Publishers, 1990.

2. Ling LH, Oh JK, Schaff HV, et al. Constrictive pericarditis in the modern era: evolving clinical spectrum and impact on outcome after pericardiectomy. Circulation 1999; 100:13801386.

3. Bertog SC, Thambidorai SK, Parakh K, et al. Constrictive pericarditis: etiology and cause specific survival after pericardiectomy. J Am Coll Cardiol 2004; 43:1445-1452.

4. Yehuda Adler $Y$, Charron $P$, Imazio $M$, et al. 2015 ESC Guidelines for the diagnosis and management of pericardial diseases. Eur Heart J 2015; 36:2921-2964.

5. Klein AL, Abbara S, Agler DA, et al. American Society of Echocardiography clinical recommendations for multimodality cardiovascular imaging of patients with pericardial disease. J Am Soc Echocardiogr 2013; 26:965-1012.

6. Cosyns B, Plein S, Nihoyanopoulos $P$, et al. European Association of Cardiovascular Imaging (EACVI) position paper: multimodality imaging in pericardial disease. Eur Heart $J$ Cardiovasc Imaging 2014; 16:12-31.
7. Bogaert J, Francone M. Pericardial disease: value of CT and MR imaging. Radiology 2013; 267:340-356.

8. Imazio M, Adler $\mathrm{Y}$. Management of pericardial effusion. Eur Heart J 2013; 34:1186-1197.

9. Talreja DR, Nishimura RA, Oh JK, Holmes DR. Constrictive pericarditis in the modern era: novel criteria for diagnosis in the cardiac catheterization laboratory. J Am Coll Cardiol 2008; 51:315-319.

10. Donadio Abduch MA, Alencar AM, Mathias W Jr, de Campos Vieira ML. Cardiac Mechanics Evaluated by Speckle Tracking Echocardiography. Arq Bras Cardiol 2014; 102(4):403-412.

11. Ufuk $Y$, Kestelli M, Yilik L, et al. Recent surgical experience in chronic constrictive pericarditis. Texas Heart Inst J 2003; 30(1):27-30.

12. Isogai $T$, Yasunaga $H$, Matsui $H$, Tanaka $H$, Hisagi M, Fushimi K. Clinical Practice Patterns in Constrictive Pericarditis Patients with Heart Failure: A Retrospective Cohort Study Using a National Inpatient Database in Japan. Clin Cardiol 2015; 38:740-746.

13. Schwefer M, Aschenbach R, Heidemann J, Mey C, Lapp H. Constrictive pericarditis, still a diagnostic challenge: comprehensive review of clinical management. Eur $J$ Cardiothoracic Surg 2009; 36:502-510. 